\title{
O Envolvimento Paterno em Famílias de Diferentes Níveis Socioeconômicos
}

ARTIGO ORIGINAL

\author{
Quele de Souza Gomes ${ }^{1}$ \\ Patrícia Alvarenga \\ Universidade Federal da Bahia
}

\begin{abstract}
RESUMO - O objetivo deste estudo foi caracterizar o envolvimento paterno em famílias de diferentes níveis socioeconômicos. Participaram 81 pais de crianças entre 4 e 6 anos, que foram classificados em três níveis socioeconômicos de acordo com a escala Hollingshead. Os pais responderam ao Questionário de Engajamento Paterno e à Ficha de Dados Sociodemográficos. Foram utilizados os testes ANCOVA e Kruskal-Wallis para verificar as diferenças no envolvimento paterno entre os grupos. Os resultados não revelaram diferenças no envolvimento paterno entre os grupos de diferentes níveis socioeconômicos, exceto na dimensão cuidados básicos. Discute-se a semelhança entre os três grupos e como as novas concepções e expectativas sobre a paternidade estão sendo compartilhadas por diferentes estratos da sociedade.
\end{abstract}

Palavras-chave: paternidade, envolvimento paterno, fatores socioculturais, nível socioeconômico

\section{Paternal Involvement in Families from Different Socioeconomic Status.}

\begin{abstract}
The aim of this study was to characterize paternal involvement in families from different socioeconomic levels. Participated 81 fathers of children between 4 and 6 years, which were classified into three socioeconomic levels, according to Hollingshead scale. Fathers responded to Paternal Engagement Questionnaire and Socio-Demographic Data Sheet. ANCOVA and Kruskal- Wallis tests were used to verify differences in paternal involvement among groups. The results revealed no differences in paternal involvement among groups from different socioeconomic levels, except in the dimension basic care. Therefore is discussed the similarity among the three groups and how new ideas and expectations about parenthood are shared by different strata of society.
\end{abstract}

Keywords: paternity, paternal involvement, sociocultural factors, socioeconomic status

Entre os séculos XVIII e XIX, pai e mãe exerciam papéis distintos, sendo a mãe a principal cuidadora e responsável pelo desenvolvimento da criança, enquanto o pai era o provedor financeiro da família. No final do século XX e início do século XXI, o papel do pai foi ampliado devido a mudanças sociais, como a inserção da mulher no mercado de trabalho e a ascensão do movimento feminista (Borsa \& Nunes, 2011; Cabrera, Fitzgerald, Bradley, \& Roggman, 2007; Marsiglio \& Pleck, 2005; Seabra \& Seidl-de-Moura, 2012).

Em virtude da participação crescente do pai nos cuidados e no convívio com os filhos e da repercussão dessas interações no desenvolvimento infantil, diferentes construtos têm sido criados para sistematizar o estudo da relação pai-criança. Entre eles, o envolvimento paterno, que pode ser definido como o conjunto de comportamentos emitidos pelo pai em relação à criança bem como os afetos, percepções e crenças que ele demonstra em relação ao seu filho (Lamb, Pleck, Charnov, \& Levine, 1985, Marsiglio \& Pleck, 2005; Pleck, 2010). Inicialmente, o envolvimento paterno era compreendido a partir de três dimensões: interação, acessibilidade e responsabilidade (Lamb et al., 1985). Mais recentemente, um novo modelo teórico com cinco dimensões, baseado em Lamb et al. (1985), foi desenvolvido por Pleck (2010).

1 Endereço para correspondência: Universidade Federal da Bahia, Rua Aristides Novis, 197, Salvador, BA, Brasil. 40210-730. E-mail: quelesg@gmail.com
Nesse novo modelo, a dimensão interação - do modelo original - passou a ser chamada engajamento positivo em atividades e refere-se à interação direta do pai com a criança, compreendendo comportamentos paternos como dar banho na criança ou alimentá-la, ajudar ou ensinar alguma habilidade, conversar ou brincar com a criança. A categoria acessibilidade - do antigo modelo - foi ampliada e denominada afeto/responsividade, dizendo respeito à capacidade do pai de prover suporte emocional para seus filhos e expressar seus sentimentos em relação a eles, demonstrando afeto através de gestos ou palavras, por exemplo. A dimensão responsabilidade foi segmentada em duas novas dimensões denominadas cuidados indiretos e processo de responsabilidade. Os cuidados indiretos dizem respeito à realização, por parte do pai, de atividades para a criança sem que ocorra a interação direta com ela e subdividese em duas subcategorias: cuidados indiretos materiais e cuidados indiretos específicos. Cuidados indiretos materiais refere-se a comportamentos paternos como comprar e organizar bens e serviços para a criança; cuidados indiretos específicos relaciona-se a condutas como escolher a escola na qual o filho estudará ou agendar uma consulta médica para ele. Por fim, a dimensão processo de responsabilidade refere-se ao comportamento de tomar a iniciativa e monitorar o comportamento, as atividades e outros aspectos da vida da criança independentemente da divisão de tarefas preestabelecida com a mãe, como oferecer cuidados básicos sem que a criança ou a mãe solicite. Além das alterações já citadas, foi adicionada ao novo modelo a dimensão controle 
para se referir ao exercício paterno de monitorar as atividades da criança, estabelecer regras de conduta, disciplinar a criança por meio de práticas não coercitivas, bem como o conhecimento do pai acerca da rotina da criança.

Tendo em vista o crescente interesse teórico frente a esse novo contexto no qual o pai passa a participar de diferentes modos da criação dos filhos, bem como as implicações dessa participação para o desenvolvimento infantil (Gomes, Crepaldi, \& Brigas, 2013; Pancsofar, Vernon-Feagans, \& Odom, 2013; Storhaug, 2013), os fatores que contribuem para a promoção do envolvimento paterno têm se tornado foco de muitas investigações. Alguns estudos têm indicado que fatores biológicos como características que foram selecionadas na história da evolução da espécie (Nunes \& Vieira, 2009; Vieira et al., 2009), características individuais paternas como personalidade, saúde mental e história de vida (Amato \& Rivera, 1999; Bossardi, Gomes, Vieira, \& Crepaldi, 2013; Gaertner, Spinrad, Eisenberg, \& Greving, 2007; Rienks, Wadsworth, Markman, Einhorn, \& Etter, 2011; Souza \& Benetti, 2008) e aspectos inerentes à criança como sexo, idade e condições de saúde (McBride, Shoppe, \& Rane, 2002; Monteiro, Veríssimo, Santos, \& Vaughn, 2008; Rienks et al., 2011; Seabra \& Seidl-Moura, 2012) afetam o envolvimento paterno. Além de variáveis filogenéticas e individuais do pai e da criança, aspectos contextuais e culturais, como, por exemplo, aqueles relacionados às políticas públicas de apoio à paternidade e ao nível socioeconômico (NSE), também parecem influenciar o quanto o pai se envolve na criação dos filhos (Amato \& Rivera, 1999; Bossardi et al., 2013; Carvalho, 2016; Monteiro et al., 2008; Souza \& Benetti, 2008).

Entre os aspectos relacionados ao NSE que podem influenciar o modo como os pais participam da criação dos filhos, destacam-se o nível de escolaridade, a ocupação e a riqueza ou renda (Bornstein \& Bradley, 2014). Em relação ao nível de escolaridade, Amato e Rivera (1999) constataram que pais com maior nível de escolaridade apresentavam maior nível de envolvimento paterno. Nessa mesma direção, Monteiro et al. (2010) concluíram que, quanto maior é a escolaridade do pai, maior o seu envolvimento em atividades de cuidados indiretos e menor o envolvimento em brincadeiras. Em contrapartida, Pimenta, Veríssimo, Monteiro e Costa (2010), Castillo, Welch e Sarver (2011) e Rienks et al. (2011) não verificaram relação entre nível de escolaridade e envolvimento paterno. Assim como as pesquisas internacionais, no Brasil, os estudos que investigaram a relação entre nível de escolaridade e envolvimento paterno também apresentaram resultados controversos. Os resultados do estudo de Souza e Benetti (2008), por exemplo, indicaram que, quanto maior era o nível de escolaridade dos pais, mais eles contribuíam ativamente no cuidado didático e mais eram afetivos e participavam mais de atividades sociais com seus filhos, quando comparados a pais com menor nível de escolaridade. Em contrapartida, Bossardi et al. (2013) verificaram que o nível de escolaridade do pai não estava correlacionado significativamente com o envolvimento paterno. A ausência de um consenso a esse respeito na literatura (Bossardi et al., 2013; Castillo et al., 2011; Pimenta et al., 2010) pode ter ocorrido em virtude da pouca variabilidade em relação às características sociodemográficas das amostras investigadas e a diferenças nos objetivos dos estudos (Rienks et al., 2011).

No que tange ao prestígio ocupacional do pai, Shows e Gerstsel (2009) compararam como dois grupos de homens vivenciavam a masculinidade. No primeiro grupo, constituído por pais considerados com maior prestígio profissional - os médicos -, os autores constataram maior envolvimento em atividades sociais e de lazer com as crianças do que em atividades de cuidado diário, enquanto os pais que representavam a classe trabalhadora - os técnicos de enfermagem -, além de envolverem-se nas atividades sociais e de lazer, envolviam-se mais do que os médicos nos cuidados diários com a criança. Em contrapartida, Cooklin et al. (2015) verificaram que o maior prestígio ocupacional do pai estava positivamente associado à participação paterna nas atividades relacionadas aos aspectos da vida familiar e criação dos filhos.

Em relação à influência da renda familiar sobre o envolvimento paterno, os achados também são controversos. No contexto brasileiro, Souza e Benetti (2008), ao investigarem o envolvimento paterno, em uma amostra de homens desempregados, verificaram que os pais que recebiam contribuição na renda familiar de outros membros da família ou de outras fontes significativas estavam mais envolvidos com seus filhos do que os pais que não recebiam nenhum tipo de contribuição, em quatro dimensões específicas do envolvimento: didática, afetiva, disponibilidade e social. Já Rienks et al. (2011), em contexto internacional, verificaram relação contrária: pais que tinham menor renda eram mais envolvidos com seus filhos. Essa divergência pode ter ocorrido porque no estudo de Souza e Benneti (2008), devido ao fato de os pais estarem desempregados e dessa condição acarretar não só uma privação de recursos materiais, mas também problemas emocionais tais como ansiedade e depressão, os pais que recebiam algum tipo de contribuição poderiam ter tido esses efeitos atenuados. Logo, isso possibilitaria que esses homens tivessem maior disponibilidade emocional e de tempo para participar da rotina da criança, o que pode não ter ocorrido com os pais que não recebiam nenhuma contribuição. Além disso, no estudo de Rienks et al. (2011), os pais cuja renda era maior apresentavam maior carga horária de trabalho quando comparados aos pais com menor renda, o que pode ter impactado negativamente na disponibilidade de tempo que os pais tinham para cuidar da criança.

A paternidade é um fenômeno complexo que se constitui a partir das práticas econômicas, sociais e culturais (Marion, Ferreira, \& Pereira, 2015). Parte dos aspectos que afetam a paternidade pode ser contemplada por meio da noção de NSE. A análise dos estudos que investigam o impacto do NSE e de variáveis relacionadas sobre a paternidade indica a presença de controvérsias nos resultados, assim como uma escassez de investigações sobre essas relações no contexto brasileiro. Considerando as desigualdades sociais presentes no contexto brasileiro e a grande parcela da população que vive em condições de vulnerabilidade socioeconômica, estudos que avaliem o impacto do nível socioeconômico sobre a paternidade podem, além de ampliar a compreensão desse fenômeno, revelar a importância de ações voltadas para o incentivo à participação do pai no desenvolvimento 
infantil junto a estratos específicos da população. Portanto, torna-se relevante investigar como o NSE, enquanto produto da interação de fatores como nível de escolaridade, ocupação e renda (Bornstein \& Bradley, 2014), influencia o modo como os pais criam seus filhos.

Desse modo, o presente estudo teve como objetivo caracterizar o envolvimento paterno em famílias de diferentes NSE. A hipótese principal foi a de que pais de NSE alto apresentariam maior nível de envolvimento paterno do que pais de NSE médio que, por sua vez, apresentariam mais envolvimento paterno do que os pais de NSE baixo.

\section{Método}

\section{Participantes}

Participaram deste estudo 81 pais de crianças de ambos os sexos, com idade entre 4 e 6 anos, residentes na cidade de Salvador. Para participar do estudo, os pais deveriam ter, no mínimo, 19 anos de idade, residir com a criança e com a mãe desta, bem como exercer uma atividade laboral. Os 81 pais foram divididos em três grupos de acordo com a escala de NSE de Hollingshead (1975): NSE baixo (NSE-B), que contou com 31 participantes; NSE médio (NSE-M), 21 participantes; e NSE alto (NSE-A), 29 participantes.

O NSE, segundo Hollingshead (1975), é obtido a partir da análise de três fatores: educação, ocupação e estado civil (Tudge \& Frizzo, 2002). Os escores gerados podem ser classificados em cinco níveis socioeconômicos: nível 1 (8-19): empregados domésticos; nível 2 (20-29): operadores de máquinas; nível 3 (30-39): auxiliares de escritório e balconistas; nível 4 (40-54): proprietários de empresas de médio porte e técnicos; e nível 5 (acima de 55): proprietários de grandes empresas e profissionais de nível superior. Para fins deste estudo, os níveis propostos na escala Hollingshead foram agrupados da seguinte forma: os níveis 4 e 5 correspondem ao NSE-A, o nível 3 corresponde ao NSE-M e os níveis 1 e 2 correspondem ao NSE-B. A amostra foi selecionada por acessibilidade, em escolas, organizações públicas e privadas da cidade de Salvador e através da técnica de bola de neve, que consistiu na indicação de outros pais pelos participantes que já haviam sido selecionados para o estudo. A Tabela 1 apresenta os dados sociodemográficos dos participantes.

Alguns dados chamam atenção por diferenciarem claramente os três NSE analisados. Em relação ao nível de escolaridade, os pais de NSE-B $(M=9,32, D P=2,70)$ tinham desde o ensino fundamental incompleto ao ensino médio completo; os pais de NSE-M $(M=13,14, D P=2,08)$ tinham desde o ensino médio completo ao nível superior completo; e os pais de NSE-A $(M=17,21, D P=2,81)$ tinham desde o ensino médio completo a pós-graduação (especialização, mestrado ou doutorado). A renda familiar ${ }^{1}$ média das famílias de NSE-B foi de $\mathrm{R} \$ 1.646,41$ ( $D P=\mathrm{R} \$ 488,66)$, variando de $\mathrm{R} \$ 700,00$ a R\$2.500,00; nas famílias de NSE-M, foi de $\mathrm{R} \$ 3.365,95$ ( $D P=\mathrm{R} \$ 1.852,49)$, variando de $\mathrm{R} \$ 999,00$ a R\$7.000,00; e, nas famílias de NSE-A, a renda média foi de $\mathrm{R} \$ 6.479,27(D P=\mathrm{R} \$ 4.982,96)$, variando de $\mathrm{R} \$ 999,00$ a $\mathrm{R} \$ 25.000,00$. Em relação à carga horária de trabalho semanal dos pais, os pais de NSE-B trabalhavam em média 50,29 horas $(D P=8,90)$; os pais de NSE-M, 53,33 horas $(D P=16,05)$; e os de NSE-A, 45,38 horas $(D P=7,61)$.

\section{Instrumentos}

Ficha de Dados Sociodemográficos. O principal objetivo desse instrumento foi obter informações detalhadas sobre a escolaridade e a ocupação de ambos os pais, a fim de se realizar a classificação do nível socioeconômico da família, de acordo com a escala de Hollingshead (1975).

Questionário de Engajamento Paterno(Questionnaire d'Engagement Paternel; QEP). É um instrumento que possibilita a caracterização do envolvimento paterno a partir do relato do pai acerca da frequência com que participa dos cuidados e atividades com a criança (Paquette, Bolté, Turcotte, Dubeau, \& Bouchard, 2000). A versão brasileira foi traduzida e adaptada por Crepaldi, Vieira, Bossardi, Gomes e Bolze (2013). O questionário é composto por 52 itens, que descrevem comportamentos emitidos pelo pai em relação à criança a partir de sete dimensões: suporte emocional, abertura ao mundo, cuidados básicos, jogos físicos, evocações, disciplina e tarefas de casa. Cada item deve ser respondido pelo pai, de acordo com a frequência com que ele realiza as atividades descritas no instrumento. Essas respostas são registradas em duas escalas Likert. O modelo teórico de envolvimento paterno utilizado neste estudo foi o de Pleck (2010), sendo necessário, portanto, realizar um emparelhamento entre as dimensões acima descritas e o modelo teórico utilizado. Desse modo, a dimensão engajamento positivo em atividades foi avaliada através das dimensões cuidados básicos e jogos físicos do QEP. A dimensão afeto/responsividade foi avaliada por meio dos itens da dimensão suporte emocional e a dimensão controle foi mensurada por meio dos itens de disciplina do QEP. Por fim, a dimensão cuidados indiretos foi avaliada por meio dos itens da dimensão tarefas de casa do instrumento. A única dimensão do modelo teórico que não foi avaliada neste estudo foi a de processo de responsabilidade, tendo em vista que os itens do QEP não avaliavam se o pai realizava atividades de cuidado e lazer em relação à criança independente da divisão de tarefas preestabelecida com a mãe. Cada uma das dimensões do instrumento gera um escore que é determinado por meio da média das respostas dos pais aos itens referentes àquela dimensão. $\mathrm{O}$ escore total de envolvimento paterno é obtido por meio de uma média das respostas dos pais aos 52 itens do instrumento. Neste estudo, o valor do alfa de Cronbach foi de 0,84 .

1 O valor do salário mínimo, no ano da coleta de dados (2014), foi de R\$ 724,00 (Brasil, 2013). Logo, a renda familiar média das famílias de NSE-B, NSE-M e NSE-A correspondeu a 2,27, 4,65 e 8,95 salários mínimos, respectivamente. 
Tabela 1. Dados Sociodemográficos dos Participantes $(N=81)$

\begin{tabular}{lccc}
\hline & NSE Baixo $(\mathbf{n}=\mathbf{3 1})$ & NSE Médio (n=21) & NSE Alto (n=29) \\
\cline { 2 - 4 } & M (DP) & M (DP) & M (DP) \\
\cline { 2 - 4 } Idade do pai & $34,45(5,94)$ & $35,67(5,21)$ & $37,52(6,62)$ \\
Número filhos & $0,90(1,10)$ & $0,81(0,81)$ & $0,93(1,30)$ \\
Escolaridade pai (anos de estudo) & $9,32(2,70)$ & $13,14(2,08)$ & $17,21(2,81)$ \\
Carga horária semanal de trabalho pai & $50,29(8,90)$ & $53,33(16,05)$ & $45,38(7,61)$ \\
Idade da mãe & $31,26(6,19)$ & $33,24(4,71)$ & $35,55(5,50)$ \\
Escolaridade mãe (anos de estudo) & $9,32(2,41)$ & $12,05(2,90)$ & $16,17(3,53)$ \\
Carga horária de trabalho semanal mãe & $40,85(11,31)$ & $48,06(16,33)$ & $34,23(11,58)$ \\
Renda familiar & $1.646,61(488,66)$ & $3.365,95(1.852,49)$ & $6.479,27(4.982,96)$ \\
Idade da criança & $5,10(0,79)$ & $4,95(0,86)$ & $4,86(0,69)$ \\
Sexo da criança & Masculino 15 & Masculino 11 & Masculino 16 \\
& Feminino 16 & Feminino 10 & Feminino 13 \\
Mãe trabalha & Sim 14 & Sim 17 17 & Sim 22 \\
& Não 17 & Não 4 & Não \\
Residentes na casa & $3,81(0,87)$ & $3,95(0,97)$ & $3,76(0,87)$ \\
\hline
\end{tabular}

\section{Delineamento e Procedimentos}

No presente estudo, foi utilizado o delineamento de grupos contrastantes (Nachimias \& Nachimias, 1996), envolvendo três grupos de pais: pais de NSE-B, pais de NSE-M e pais de NSE-A. Os pais foram convidados para participar da pesquisa nas escolas em que seus filhos estudavam e nas empresas ou organizações em que trabalhavam, além de terem sido indicados por outros pais que já haviam sido incluídos na amostra. Nesses casos em que houve indicação, o contato inicial foi feito por telefone. Os participantes que foram recrutados em empresas de ônibus, canteiros de obras e limpeza pública foram incluídos no grupo NSE-B. Os participantes que compõem o NSE-M foram contatados em escolas particulares de educação infantil e instituições militares, e os participantes de NSE-A foram contatados em empresas de grande porte, faculdades ou universidades.

Os pais que foram contatados em escolas de educação infantil receberam o convite para participar da pesquisa por meio da agenda escolar da criança e, caso tivessem interesse em participar, informavam seu nome e telefone. Tanto os pais que foram indicados por outros participantes quanto os pais que informavam seu nome e telefone através da agenda escolar da criança receberam uma ligação da pesquisadora que, nesse contato, agendava a entrevista com o pai em sua residência ou em seu local de trabalho, conforme a disponibilidade do participante. Os demais pais que foram convidados para participar da pesquisa em seus locais de trabalho e consentiram em participar do estudo foram entrevistados em uma sala previamente selecionada pelos gestores organizacionais com esse objetivo. Todos os pais que concordaram em participar da pesquisa, inicialmente, assinaram o Termo de Consentimento Livre e Esclarecido e, posteriormente, responderam à Ficha de Dados Sociodemográficos e ao QEP (versão original ProsPère citado por Bossardi et al., 2013 e adaptado por Crepaldi et al., 2013). Todos os instrumentos foram aplicados em forma de entrevista, isto é, a pesquisadora lia os itens contidos nos instrumentos para os participantes que, com o auxílio de cartões de resposta, indicavam a alternativa escolhida. Este estudo foi aprovado pelo Comitê de Ética da Escola de Enfermagem da Universidade Federal da Bahia, CAAE 26960014.2.0000.5531, parecer 632.503 e está de acordo com as normas de pesquisa envolvendo seres humanos.

Tabela 2. Média e Desvio-padrão dos Escores das Dimensões de Envolvimento Paterno nos Três Niveis Socioeconômicos, valores de F e p (ANCOVA).

\begin{tabular}{|c|c|c|c|c|c|}
\hline \multirow[t]{2}{*}{ Dimensões } & NSE Baixo & NSE Médio & NSE Alto & \multirow[t]{2}{*}{$\mathrm{F}$} & \multirow[t]{2}{*}{$\mathrm{p}$} \\
\hline & $\mathrm{M}(\mathrm{DP})$ & $\mathrm{M}(\mathrm{DP})$ & $\mathrm{M}(\mathrm{DP})$ & & \\
\hline Abertura ao mundo & $3,51(0,72)$ & $3,51(0,70)$ & $3,70(0,57)$ & 0,73 & 0,43 \\
\hline Suporte emocional & $4,66(0,38)$ & $4,62(0,37)$ & $4,59(0,32)$ & 0,72 & 0,91 \\
\hline Disciplina & $4,29(0,71)$ & $4,48(0,55)$ & $4,46(0,53)$ & 2,10 & 0,51 \\
\hline Tarefas de casa & $3,32(0,50)$ & $3,21(0,66)$ & $3,25(0,61)$ & 2,18 & 0,11 \\
\hline Evocações & $4,00(0,87)$ & $4,14(0,71)$ & $4,13(0,64)$ & 2,96 & 0,89 \\
\hline Escore total QEP & $4,25(0,50)$ & $4,41(0,43)$ & $4,45(0,39)$ & 1,29 & 0,34 \\
\hline
\end{tabular}




\section{Resultados}

Para examinar as diferenças no envolvimento paterno entre os três grupos no escore total do QEP e nas dimensões abertura ao mundo, suporte emocional, disciplina, tarefas de casa e evocações foi utilizada a ANCOVA. A Tabela 2 apresenta esses resultados.

Conforme a Tabela 2, não foram observadas diferenças entre os grupos no que tange às dimensões abertura ao mundo $[F(2,78)=0,73, p=0,43]$; suporte emocional $[F(2,78)=0,72$, $p=0,91]$; disciplina $[F(2,78)=2,10, p=0,51]$; tarefas de casa $[F(2,78)=2,18, p=0,61]$; evocações $[F(2,78)=2,96, p=0,89]$ e escore total do QEP $[F(2,78)=1,29, p=0,34]$

A Tabela 3, por sua vez, apresenta os resultados do teste de Kruskal-Wallis, alternativa não paramétrica à ANOVA, utilizado para verificar as diferenças no envolvimento paterno nas dimensões cuidados básicos e jogos físicos.

Os resultados dos testes de Mann-Whitney, conforme a Tabela 4, indicaram que não foram encontradas diferenças em relação à dimensão cuidados básicos entre os pais de NSE-B e NSE-M $(U=239, z=-1,61, p=0,10)$ e entre os pais do NSE-M e NSE-A $(U=266,50, z=-0,20, p=0,45)$. Não obstante, entre os pais de NSE-B e NSE-A foram encontradas diferenças em relação a essa dimensão, indicando que os pais de NSE-A relataram realizar mais atividades - dar de comer ou beber ao filho, banhar ou vestir a criança, bem como levála ao médico, se necessário - do que os pais de NSE-B ( $U=$ $276,50, z=-2,55 ; p=0,010)$.

\section{Discussão}

O objetivo deste estudo foi caracterizar o envolvimento paterno em famílias de diferentes NSE. A hipótese foi a de que os pais de NSE-A apresentariam maior nível de envolvimento paterno do que os pais de NSE-M, que, por sua vez, apresentariam mais envolvimento paterno do que os pais de NSE-B. Essa hipótese recebeu pouco apoio dos resultados, já que os pais dos três níveis socioeconômicos fizeram relatos muito semelhantes em relação às dimensões que caracterizaram o envolvimento paterno, exceto na categoria cuidados básicos.

A semelhança no envolvimento paterno entre os grupos nas dimensões engajamento positivo em atividades (jogos físicos) e controle se constituem, historicamente, como comportamentos inerentes ao pai, pois se referem ao modelo tradicional e moderno de paternidade, cujas principais características referem-se às funções paternas de prover o lar e disciplinar a criança, assim como brincar com ela (Bueno, Gomes, \& Crepaldi, 2015; Vieira et al., 2014).

Em contrapartida, a ausência de diferenças nos relatos paternos sobre as atividades inerentes às dimensões afeto/ responsividade e cuidados indiretos pode estar relacionada às expectativas mais recentes quanto à paternidade ou ao modelo de pai cogenitor. Nesse contexto, espera-se que ocorra maior igualdade de responsabilidade entre homens e mulheres em relação à criação dos filhos, o que implica a participação ativa do pai em tarefas que viabilizem o acesso a recursos emocionais e materiais necessários ao bem-estar da criança e da família (Bueno et al., 2015; Vieira et al., 2014).

Tabela 3. Mediana e Desvio-padrão dos Escores das Dimensões Cuidados Básicos e Jogos Físicos de Envolvimento Paterno nos Três Níveis Socioeconômicos, valores de F e p (Kruskal-Wallis).

\begin{tabular}{lllllll}
\hline \multirow{2}{*}{ Dimensões } & NSE Baixo & NSE Médio & NSE Alto & \multirow{2}{*}{ F } & \multirow{2}{*}{ p } \\
\cline { 2 - 3 } & $\boldsymbol{M}(\boldsymbol{D P})$ & $\boldsymbol{M}(\boldsymbol{D P})$ & $\boldsymbol{M}(\boldsymbol{D} \boldsymbol{P})$ & & & \\
\hline \multirow{3}{*}{ Cuidados básicos } & $3,38(0,94)$ & $3,79(0,72)$ & $3,98(0,45)$ & 2 & 0,03 \\
Jogos físicos & $3,37(1,19)$ & $3,82(0,89)$ & $3,79(0,73)$ & 2 & 0,34 \\
& & & & & \\
\hline
\end{tabular}

Tabela 4. Mediana e Desvio-padrão do Escore da dimensão Cuidados Básicos de Envolvimento Paterno nos NSE Baixo e Médio, Médio e Alto, Baixo e Alto e valores de U e p (Teste MannWhitney), respectivamente.

\begin{tabular}{lcccc}
\hline \multirow{2}{*}{ Dimensão } & NSE da família & Mediana & \multirow{2}{*}{ U } & p \\
\cline { 2 - 3 } & & Md (DP) & & 0,10 \\
& Baixo & $3,38(0,94)$ & 239,00 & \\
& Médio & $3,68(0,72)$ & & 0,45 \\
$\begin{array}{l}\text { Cuidados } \\
\text { básicos }\end{array}$ & Médio & $3,68(0,72)$ & 266,50 & \\
& Alto & $3,94(0,95)$ & & \\
& Baixo & $3,38(0,94)$ & 276,50 & 0,01 \\
& Alto & $3,94(0,95)$ & & \\
\hline
\end{tabular}


Logo, as semelhanças verificadas neste estudo no que tange às dimensões do envolvimento paterno supracitadas podem ser explicadas a partir da interação de características individuais do pai e fatores sociais e políticos que visam a apoiar a participação do pai na criação dos filhos (Bossardi, Gomes, Bolze, Crepaldi, \& Vieira, 2016; Bueno et al., 2015; Carvalho, 2016; Gomes, 2016; Marion et al., 2015; Vieira et al., 2014).

Entre as características individuais paternas, apesar de ainda não existir consenso na literatura acerca da influência da idade do pai sobre o seu envolvimento com a criança, Amato e Rivera (1999), Perry, Harmon e Lepp (2011) e Monteiro et al. (2010) constataram que pais mais jovens são mais envolvidos com suas crianças do que pais mais velhos. Desse modo, é possível inferir que um dos fatores que pode justificar a semelhança nos relatos dos participantes sobre o envolvimento paterno é a idade dos pais, já que os participantes do presente estudo tinham idades semelhantes e, de acordo com Papalia, Feldman e Olds (2013), pode-se considerar que ainda estão no início da vida adulta.

Ainda com relação à idade do pai, é válido ressaltar que a maioria dos participantes nasceu a partir da década de 1970, período que, de acordo com Bossardi et al. (2016), coincide com a ascensão de um novo modelo econômico industrial e uma maior participação das mulheres no mercado de trabalho, situação que pode ter contribuído para que os pais dos participantes deste estudo estivessem mais envolvidos com os filhos, visto que foi justamente nesse período que a mulher deixou de dedicar-se exclusivamente às atividades ligadas à família e ao lar. Por conseguinte, tal mudança pode ter contribuído para que os entrevistados deste estudo fossem criados por pais que transitavam entre os modelos tradicional e moderno de paternidade.

Além dos fatores relacionados à idade do pai, variáveis culturais e sociais como o incentivo à participação do homem na criação do filho assim como o reconhecimento de que o pai é importante para o desenvolvimento infantil podem ter contribuído para que os pais participassem mais da criação dos filhos. Nesse sentido, certas intervenções do Estado na vida familiar também podem ter repercutido sobre o exercício da paternidade (Bueno et al.; Carvalho, 2016). São exemplos dessas medidas o Estatuto da Criança e do Adolescente (Brasil, 1990), a Política Nacional de Atenção Integral à Saúde do Homem (Brasil, 2009) e a instituição da guarda compartilhada (Schneebeli \& Menandro, 2014).

De qualquer modo, é importante considerar a diferença encontrada entre os pais de NSE-B e NSE-A no que tange à sua participação em atividades relacionadas à dimensão engajamento positivo em atividades, particularmente nos cuidados básicos. As atividades inerentes à dimensão cuidados básicos referem-se à participação paterna em funções histórica e socialmente consideradas como sendo exclusivas da mãe (Vieira et al., 2014), o que pode nos levar a supor que os pais de NSE-A estão cada vez mais propensos a participarem dos cuidados diretos da criança, seja porque sua companheira está trabalhando, seja porque não desejam reproduzir o mesmo padrão ao qual foram expostos durante a infância (Prado \& Abrão, 2015). Além disso, é possível que os pais de NSE-A, conforme os resultados deste estudo, sejam aqueles que estão mais predispostos a se envolverem em atividades relacionadas aos cuidados básicos da criança em virtude de possuírem uma carga horária de trabalho semanal inferior à dos pais de NSE-B, o que possibilitaria a eles estarem mais presentes no cotidiano dos filhos (Pimenta et al., 2010).

Outro fator que pode ter contribuído para que os pais de NSE-A estivessem mais envolvidos em atividades de cuidados básicos com a criança do que os pais de NSE-B refere-se ao status ocupacional da mãe e ao nível de escolaridade de ambos os pais, aspectos relacionados ao NSE da família. No grupo de pais de NSE-A, por exemplo, mais da metade das mães trabalhava, enquanto que, no grupo de pais de NSE-B, a relação foi contrária, isto é, a maioria das mães não trabalhava. Apesar de não terem sido verificadas diferenças quanto à carga horária de trabalho semanal das mães, é possível conjeturar que, devido ao fato de a mãe estar em casa, o pai poderia ser menos solicitado a participar dos cuidados diretos com a criança, já que a mãe estaria disponível para realizar essas atividades.

Ainda nessa perspectiva, observou-se que as mães e os pais de NSE-A apresentavam mais anos de escolaridade do que os pais de NSE-B. Em relação à influência da escolaridade materna sobre o modo como o pai participa da criação dos filhos, supõe-se que, à medida que a mãe se qualifica, ela tem mais chances de ingressar no mercado de trabalho, sendo, desse modo, necessário que o pai participe mais ativamente dos cuidados com a criança, já que a mesma não pode ficar desassistida (Arruda \& Lima, 2013). Além disso, o acesso à informação por parte dessas mulheres pode instrumentalizálas a exigir mais a participação do parceiro nesse tipo de atividade. England e Srivastava (2013) constataram que o maior nível de escolaridade da mãe estava associado à maior participação do pai nos cuidados com a criança. Essas autoras argumentaram que o fato de o pai participar mais das atividades com os filhos pode não ocorrer em virtude do nível de escolaridade dele próprio, mas pelo fato de que, sendo a mãe mais escolarizada, ela poderia exigir dele uma participação mais intensa. No contexto internacional, por exemplo, pesquisas têm associado o nível de escolaridade do pai e da mãe ao maior envolvimento dos pais em atividades de cuidado com os filhos (England \& Srivastava 2013; Reich, 2014). Apesar de os achados do presente estudo somente indicarem diferenças entre os grupos no que tange à participação do pai em atividades de cuidados básicos, é possível que essa diferença, de fato, deva-se em parte à maior escolaridade dos pais (Cabrera \& Bradley, 2012).

Ainda em relação às diferenças encontradas entre os pais de NSE-B e NSE-A quanto à participação em atividades de cuidados básicos, é possível argumentar que, além dos fatores já citados, aspectos relacionados à renda e à cultura podem influenciar o modo como os pais participam da criação dos filhos. De acordo com Marion et al. (2015), famílias de camadas mais populares tendem a organizar os cuidados com os filhos a partir da rede familiar extensa (tios e avós) e isso inclui responsabilizar as mulheres da família pelos cuidados diretos com a criança, o que contribui para que o pai seja menos solicitado a realizar esse tipo de atividade com os filhos, prevalecendo, nesse contexto, a divisão sexual do trabalho doméstico. Desse modo, é possível que os pais que pertencem ao NSE-B possuam crenças ligadas à divisão 
sexual do trabalho doméstico, o que, por sua vez, os impediria de investir tempo e esforço nesse tipo de atividade, legando à mãe a responsabilidade pelos cuidados básicos com a criança, enquanto os homens exerceriam atividades mais ligadas ao convívio social e ao lazer da criança.

Corroborando essa hipótese, Bustamante e Trad (2005), por exemplo, constataram que os homens de camadas mais populares ainda entendem o cuidado em relação à criança como uma atribuição feminina, sendo a sua principal função prover financeiramente a família. Outros estudos recentes realizados com amostras de diferentes NSE indicaram que expectativas semelhantes àquelas reportadas por Bustamante e Trad (2005) em relação ao papel do pai e da mãe ainda são comuns no Brasil (Drago \& Menandro, 2014; Prado \& Abrão, 2015).

Ainda em relação à divisão sexual do trabalho doméstico ora descrita, Marques (2015) aponta que a licençapaternidade configura-se como um direito do trabalhador que visa a manter tal estereótipo, já que, no Brasil, a licençapaternidade, quando comparada à licença-maternidade, é muito menor e não implica concessão de benefício econômico para o pai. Logo, é possível constatar que, apesar das políticas públicas brasileiras de apoio à paternidade, esse tema ainda tem pouca relevância em nosso país, tendo em vista que, em alguns países da América do Norte, da Europa e da Ásia, já não se utiliza o termo licença-paternidade ou maternidade. $\mathrm{Na}$ Suécia, por exemplo, a licença-parental foi regulamentada em 1974 e tem como objetivo promover o envolvimento de ambos os genitores, com auxílio financeiro do Estado, nos cuidados e desenvolvimento biopsicossocial da criança (Silva, 2015).

Entre as limitações inerentes a esta investigação, podemos citar a quantidade de participantes, a dificuldade em se recrutar pais que atendessem aos critérios da amostra devido à sua baixa adesão a estudos como este, o que pode ser decorrente de inúmeros fatores, tais como carga horária de trabalho e pouca familiaridade com pesquisadores. Ademais, vale ressaltar que, no contexto brasileiro, ainda não existem instrumentos validados para investigar a relação pai-criança, o que pode dificultar a avaliação desse fenômeno na nossa cultura.

\section{Considerações Finais}

Os resultados do presente estudo indicaram que o NSE da família mostrou-se relacionado apenas à dimensão dos cuidados básicos paternos em relação à criança. A comparação entre os grupos indicou que os pais de NSE-A se envolviam mais em atividades como alimentar, dar banho e vestir a criança, quando comparados aos pais de NSE-B. Nesse sentido, ressalta-se a importância de se continuar investigando o papel de variáveis como escolaridade, ocupação e renda sobre a participação do pai em cuidados básicos. Por outro lado, o pequeno impacto do NSE sobre o envolvimento paterno indica a necessidade de explorar outras variáveis, como as crenças e mecanismos culturais, que podem mediar as diferentes formas com que os homens têm vivenciado novas atribuições no contexto familiar.

\section{Referências}

Amato, P. R., \& Rivera, F. (1999). Paternal involvement and children's behavior problems. Journal of Marriage and the Family, 61(2), 375-384.

Arruda, S. L. S., \& Lima, M. C. F. (2013). O novo lugar do pai como cuidador da criança. Estudos Interdisciplinares em Psicologia, 4(2), 201-216. doi: 10.5433/2236-6407.2013v4n2p201

Bornstein, M. H., \& Bradley, R. H. (2014). Socioeconomic status, parenting, and child development: An introduction. In M. H. Bornstein \& R. H. Bradley (Eds.), Socioeconomic Status, Parenting, and Child Development (pp. 1-10). New York: Routledge.

Borsa, J. C., \& Nunes, M. L. T. (2011). Aspectos psicossociais da parentalidade: O papel de homens e mulheres na família nuclear. Psicologia Argumento, 29(64), 31-39.

Bossardi, C. N., Gomes, L. B., Vieira, M. L., \& Crepaldi, M. A. (2013). Engajamento paterno no cuidado a crianças de 4 a 6 anos. Psicologia Argumento, 31(73), 237-246.

Bossardi, C. N., Gomes, L. B., Bolze, S. D. A., Crepaldi, M. A., \& Vieira, M. L. (2016). Desafios de ser pai em uma sociedade em transformação. In L. V. de C. Moreira, E. P. Rabinovich, \& P. C. S. do V. Zucoloto (Eds.), Paternidade na sociedade contemporânea: $O$ envolvimento paterno e as mudanças na família. (pp. 81-100). Curitiba: Juruá.

Bueno, R. K., Gomes, L. B., \& Crepaldi, M. A. (2015). Desafios de ser pai em uma sociedade em transformação. In E. R. Goetz \& M. L. Vieira (Eds.), Novo pai: Recursos, desafios e possibilidades. (pp. 95-107). Curitiba: Juruá.

Bustamante, V., \& Trad, L. A. B. (2005). Participação paterna no cuidado de crianças pequenas: Um estudo etnográfico com famílias de camadas populares. Cadernos de Saúde Pública, 21(6), 1865-1874.

Brasil. (1990). Lei n. 8.069, de 13 de julho de 1990. Dispõe sobre o Estatuto da Criança e do Adolescente e dá outras providências. Diário Oficial da União, República Federativa do Brasil, Brasília, DF.

Brasil. (2009). Política Nacional de Atenção Integral à Saúde do Homem. Diário Oficial da República Federativa do Brasil, Brasília.

Brasil, (2013, Dezembro 23). Valor do salário mínimo vai para $R \$$ 724 em 2014. Portal Brasil. Retirado de http:/www.brasil.gov. br/economia-e-emprego/2013/12/valor-do-salario-minimo-vaipara-r-724-em-2014

Cabrera, N. J., Fitzgerald, H. E., Bradley, R. H., \& Roggman, L. (2007). Modeling the dynamics of paternal influences on children over the life course. Applied Development Science, 11(4), 185-189. doi: http://dx.doi.org/10.1080/10888690701762027

Cabrera, N. J., \& Bradley, R. H. (2012). Latino fathers and their children. Child Development Perspectives, 6(3), 232-238. doi: 10.1111/j.1750-8606.2012.00249.x

Carvalho, A. B. (2016). Políticas de apoio à família e à paternidade: Uma visão comparada entre as políticas norte-americanas e brasileiras. In L. V. de C. Moreira, E. P. Rabinovich, \& P. C. S. do V. Zucoloto (Eds.), Paternidade na sociedade contemporânea: $O$ envolvimento paterno e as mudanças na familia. (pp. 315-333). Curitiba: Juruá. 
Castillo, J., Welch, G., \& Sarver, C. (2011). Fathering: The relationship between fathers' residence, fathers' sociodemographic characteristics, and father involvement. Maternal and Child Health Journal, 15(8), 1342-1349. doi: 10.1007/s10995-010-0684-6

Cooklin, A. R., Giallo, R., Strazdins, 1., Martin, A., Leach, L. S.,\& Nicholson, J. M. (2015). What matters for working fathers? Job characteristics, work-family conflict and enrichment, and fathers' postpartum mental health in an Australian cohort. Social Science \& Medicine I, 146, 214-222. doi: http://dx.doi. org/10.1016/j.socscimed.2015.09.028

Crepaldi, M. A., Vieira, M. L., Bossardi, C. N., Gomes, L. B., \& Bolze, S. D. A. (2013). Questionário de Engajamento Paterno (Manuscrito não publicado). Universidade Federal de Santa Catarina, Florianópolis.

Drago, A. B., \& Menandro, M. C. S. (2014). A paternidade e a maternidade sob o olhar de jovens de classe média e baixa: Um estudo em representações sociais. Revista Colombiana de Psicologia, 22(2). 331-324. doi: https://doi.org/10.15446/ rcp.v23n2.40672

England, P., \& Srivastava, A. (2013). Educational diferences in US parents' time spent in child care: The role of culture and cross-spouse influence. Social Science Research, 42, 971-986. doi: 10.1016/j.ssresearch.2013.03.003

Gaertner, B. M., Spinrad, T. L., Eisenberg, N., \& Greving, K. A. (2007). Parental childrearing attitudes as correlates of father involvement during infancy. Journal of Marriage and Family, 69, 962-976. doi: 10.1111/j.1741-3737.2007.00424

Gomes, L. B., Crepaldi, M. A., \& Bigras, M. (2013). O engajamento paterno como fator de regulação da agressividade em pré-escolares. Paidéia, 23(54), 21-29. doi: http://dx.doi. org/10.1590/1982-43272354201304

Hollingshead, A. B. (1975). Four factor index of social status (Manuscrito não publicado). Universidade Federal do Rio Grande do Sul, Porto Alegre.

Lamb, M. E., Pleck, J. H., Charnov, E. L., \& Levine, J. A. (1985). Paternal Behavior in Humans. American Zoologist, 25(3), 883-894. doi: 10.1093/icb/25.3.883

Marion, J., Ferreira, M., \& Pereira, C. R. R. (2015). Desafios de ser pai em uma sociedade em transformação. In E. R. Goetz \& M. L. Vieira (Eds.), Novo pai: Recursos, desafios e possibilidades. (pp. 171-180). Curitiba: Juruá.

Marques, S. S. (2015). Ampliar a licença-paternidade para despatriarcalizar o Estado e a sociedade. Gênero \& Direito, 1, 241-260. doi: 10.18351/2179-7137/ged.2015n1p241-260

Marsiglio, W., \& Pleck, J. H. (2005). Fatherhood and masculinities. In M. Kimmel, J. Hearn, \& R.W. Connell,(Eds.), The handbook of studies on men and masculinities (pp. 249-269). Thousand Oaks, CA: Sage. doi: http://dx.doi.org/10.4135/9781452233833.n15

McBride, B. A., Schoppe, S. J.,\& Rane, T. R. (2002). Child characteristics, parenting stress, and parental involvement: Fathers versus mothers. Journal of Marriage and Family, 64, 998-1011. doi: 10.1111/j.1741-3737.2002.00998.x

Monteiro, L., Veríssimo, M., Santos, A. J., \& Vaughn, B. E. (2008). Envolvimento paterno e organização dos comportamentos de base segura das crianças em famílias portuguesas. Análise Psicológica, 3(26), 395-409.
Monteiro, L., Fernandes, M., Veríssimo, M., Costa, I. P., Torres, N. \&, Vaughn, B. E. (2010). Perspectiva do pai acerca do seu envolvimento em famílias nucleares. Associações com o que é desejado pela mãe e com as características da criança. Revista Interamericana de Psicologia, 44(1), 120-130.

Nachmias, C., \& Nachmias, D. (1996). Research methods in the social sciences. London: Arnold.

Nunes, S. A. N., \& Vieira, M. L. (2009). Fundamentos históricos e epistemológicos do comportamento paterno. Psicologia Argumento, 27(57), 103-115.

Pancsofar, N. Vernon-Feagans, L, \& Odom, E. C. (2013). Work characteristics and fathers' vocabulary to infants in African American families. Journal of Applied Developmental Psychology, 34(2): 73-81. doi: 10.1016/j.appdev.2012.11.005

Papalia, D. E., Feldman, R. D., \&, Olds, R. D. (2013). Desenvolvimento Humano. Porto Alegre: Artmed.

Paquette, D., Bolté, C., Turcotte, G., Dubeau, D., \& Bouchard, C. (2000). A new typology of fathering: Defining and associated variables. Infant and Child Development, 9(4), 213-230. doi: 10.1002/1522-7219(200012)9:4<213::AIDICD233>3.0.CO;2-0

Perry, A. R., Harmon, D. K., \& Leeper, J. (2011). Resident black father's involvment: A comparative analysis of married and unwed, cohabitating fathers. Journal of Family Issues, 33(6), 695-714. doi: 10.1177/0192513X11428125

Pimenta, M., Veríssimo, M., Monteiro, L., \& Costa, I. P. (2010). O envolvimento paterno de crianças a frequentar o jardim-deinfância. Análise Psicológica, 4(28), 565-580.

Prado, J. C., \& Abrão, J. L. F. (2015). Paternidade: Um estudo sobre pesquisas desenvolvidas no contexto brasileiro. Colloquium Humanarum, 11(1), 94-112.

Pleck, J. H. (2010). Paternal Involvment: Revised conceptualization and theoretical linkages with child outcomes. In T. Lamb (Ed), The role of the father in child development (pp. 67-107, 5th ed.) New York: Wiley.

Reich, N. (2014). Fathers' childcare: The difference between participation and amount of time. Journal of Family and Economic Issues, 35(2), 190-213. doi: 10.1007/s10834-0139359-y

Rienks, S. L., Wadsworth, M. E., Markman, H. J., Einhorn, L., \& Etter, E. M. (2011). Father involvement in urban low-income fathers: baseline associations and changes resulting from preventive intervention. Family Relations, 60(2), 191-204. doi: 10.1111/j.1741-3729.2010.00642.x

Seabra, K. S., \& Seidl-de-Moura, M. L. (2012). Cuidados paternos nos primeiros três anos de vida de seus filhos: Um estudo longitudinal. Interação em Psicologia, 15(2), 135-147.

Schneebeli, F. C. F., \& Menandro, M. C. S. (2014). Com quem as crianças ficarão?: Representações sociais da guarda dos filhos após a separação conjugal. Psicologia \& Sociedade, 26(1), 175-184. doi: http://dx.doi.org/10.1590/S010271822014000100019

Shows, C., \& Gertsel, N. (2009). Fathering, class, and gender: A comparison of physicians and emergency medical technicians. Gender \& Society, 23(2), 161-187. 
Silva, A. L. C. (2015). Da licença, eu sou pai! A produção de sentidos sobre a licença-paternidade com trabalhadorespais em Suape (Dissertação de Mestrado). Universidade Federal de Pernambuco, Recife. Retirado de http:// repositorio.ufpe.br/bitstream/handle/123456789/14093/ DISSERTA $\%$ C3\%87\%C3\%83O $\% 20$ Ana $\% 20$ Luisa $\% 20$ Cataldo $\% 20 \mathrm{da} \% 20$ Silva.pdf? sequence $=1 \&$ isAllowed $=\mathrm{y}$

Souza, C. L. C., \& Benetti, S. P. C. (2008). Paternidade e desemprego: Características do envolvimento paterno e aspectos do relacionamento familiar. Contextos Clínicos, $1(2), 61-71$.

Storhaug, A. S. (2013). Fathers' involvement with the Child Welfare Service, Children and Youth Services Review, 35, (10), 17511759. DOI: http://dx.doi.org/10.1016/j.childyouth.2013.08.006
Tudge, J. R. H., \& Frizzo, G.F. (2002). Classificação baseada em Hollingshead do nível socioeconômico das famílias do estudo longitudinal de Porto Alegre: da gestação à escola (Manuscrito não publicado). Universidade Federal do Rio Grande do Sul, Porto Alegre.

Vieira, M. L., Bossardi, C. N., Gomes, L. B., Bolze, S. D. A., Crepaldi, M. A., \& Piccinini, C. A. (2014). Paternidade no Brasil: Revisão sistemática de artigos empíricos. Arquivos Brasileiros de Psicologia, 66(2), 36-52.

Vieira, M. L, Rímoli, A. O., Prado, A. B., \& Chelini, M.O. (2009). Cuidado e responsividade parentais: Uma análise a partir da Teoria da História de Vida e da Teoria do Investimento Parental. In M. E. Y. Emma Otta (Org.), Psicologia evolucionista (pp. 86-95). Rio de Janeiro: Guanabara Koogan.

Recebido em 17.03.2016

Primeira decisão editorial em 15.06.2016

Versão final em 12.07.2016

Aceito em 12.08.2016 